\title{
Third-Order Susceptibility of Silicon Crystals Measured with Millimeter-Wave Gyrotron
}

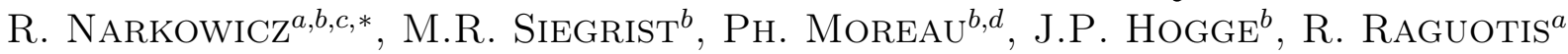 \\ AND R. BRAZIS ${ }^{a}$ \\ ${ }^{a}$ Semiconductor Phys. Institute, Center for Physical Sciences and Technology
}

A. Goštauto 11, LT-01108, Vilnius, Lithuania

${ }^{b}$ Centre de Recherches en Physique des Plasmas, Ecole Polytechnique Fédérale de Lausanne

CH-1015 Lausanne, Switzerland

${ }^{c}$ TU Dortmund University, Otto-Hahn-Str. 4, D-44221 Dortmund, Germany

${ }^{d}$ Association EURATOM-CEA CEA/DSM/IRFM, CEA-Cadarache, F-13108 Saint Paul-les-Durance, France

We investigate experimental dependence of the third harmonic generation efficiency in the $n$-type Si crystals on the geometrical dimensions of the sample, polarization and power of the fundamental wave. The efficiency increases monotonically with the rise of the sample thickness up to a threshold value, and decreases dramatically above the threshold. At shorter propagation distances the generation efficiency could be correctly simulated using the layered medium approximation and the numerically calculated electron drift velocity response to the pumping wave electric field to describe the change of the semiconductor properties under high-power microwave irradiation.

PACS: $42.65 . \mathrm{Ky}, 72.20 . \mathrm{Ht}$

\section{Introduction}

Measurements of the three-photon quantum correlation attracted recently considerable interest to the third-order nonlinear interactions, like third harmonic generation (THG) or triple photon generation in optical experiments. Direct generation experiment is difficult, because in optical media the 3rd order nonlinear susceptibility $\chi_{3}$ is several orders of magnitude lower than the second-order one, as well as due to the phase matching constraints. Only a few THG experiments were performed: in the noncentrosymmetrical crystals, where the second order nonlinearity has been suppressed along the selected axis $[1,2]$, and in the rutile crystal with the inversion center, where the 2 nd order effects vanish due to the crystal symmetry [3]. THG efficiency up to $2.5 \%$ has been achieved in these measurements. In the terahertz $(\mathrm{THz})$ range that is in between the far-infrared and millimeter-wave ranges, the high-power wave harmonic generation is difficult because of the large-scale aperture of quasi-optical nonlinear samples needed for low diffraction loss. Primary experimental investigations of the THG in quasi-optical systems have been carried out in $n$-type silicon revealing the efficiency of about $0.1 \%$ [4-6]. The causes of this rather low efficiency have not been explored. The 3rd order susceptibility values estimated in particular experiments have been found to be quite different challenging for additional experiments.

It has been suspected that the pump wave self-action can change the spatial structure of fundamental wave and the 3rd harmonic wave beams preventing the THG efficiency optimization.

\footnotetext{
* corresponding author; e-mail:

rysard.narkovic@uni-dortmund.de
}

The nonlinear optical properties of doped semiconductors in the far-infrared and millimeter wave range are determined by the response of the free-carrier system to the electric field of the incident wave. Therefore THG experiments are well suited to study high-field nonlinear transport phenomena in semiconductors.

This encouraged us to undertake new theoretical modeling and experiments on THG in silicon crystals pumped by high-power gyrotron, aiming to reveal the THG controlling factors.

\section{Theory}

Third harmonic generation in the uniform medium is described by the nonlinear wave equation, which in the case of negligible influence of the third harmonic electric field on the nonlinear polarization [7] is

$$
\nabla^{2} \boldsymbol{E}_{1,3}-\frac{\varepsilon_{1,3}}{c^{2}} \frac{\partial^{2} \boldsymbol{E}_{1,3}}{\partial t^{2}}=\frac{1}{\varepsilon_{0} c^{2}} \frac{\partial^{2} \boldsymbol{P}_{\mathrm{NL}}^{(1,3)}}{\partial t^{2}} .
$$

Actually, the right side of Eq. (1) is set to zero because the nonlinear polarization $\boldsymbol{P}_{\mathrm{NL}}^{(1)}$ at the fundamental frequency leads to the renormalization of the dielectric permittivity $\varepsilon_{1}$. The nonlinear polarization at the $3 \mathrm{rd}$ harmonic is

$$
\boldsymbol{P}_{\mathrm{NL}}^{(3)}=\varepsilon_{0} \chi_{3} E_{1}^{2} \boldsymbol{E}_{1} .
$$

The 3rd order nonlinear susceptibility and the dielectric permittivity values are $[8,9]$ :

$$
\begin{aligned}
& \chi_{3}=\frac{N e}{\omega_{1} \varepsilon_{0} E_{a 1}^{2}} \frac{V_{a 3}}{E_{a 1}} \exp \left(-\mathrm{i} \phi_{3}\right), \\
& \varepsilon_{1}=\varepsilon_{\mathrm{L}}-\mathrm{i} \frac{N e}{\omega \varepsilon_{0}} \frac{V_{a 1}}{E_{a 1}} \exp \left(\mathrm{i} \phi_{1}\right), \\
& \varepsilon_{3} \approx \varepsilon_{\mathrm{L}}-\mathrm{i} \frac{N e}{3 \omega \varepsilon_{0}} \frac{V_{a 1}}{E_{a 1}} \frac{1-\mathrm{i} \tan \phi_{1}}{1-\mathrm{i} 3 \tan \phi_{1}} \exp \left(\mathrm{i} \phi_{1}\right) .
\end{aligned}
$$

Here $\varepsilon_{\mathrm{L}}$ is lattice dielectric constant, $N$ is the electron density, $V_{a 1}, V_{a 3}, \phi_{1}, \phi_{3}$ are the 1st and 3rd harmonic 
drift velocity amplitudes and phases at the frequencies $\omega_{1}$ and $\omega_{3}$ respectively, $E_{a 1}$ is the fundamental wave electric field amplitude. The drift velocity amplitudes and phases are calculated using Monte Carlo code [10].

For the electron motion in $n$-type $\mathrm{Si}$ we consider six ellipsoidal nonparabolic X-valleys with the longitudinal and transversal masses of $0.98 m_{0}$ and $0.19 m_{0}$ and the band nonparabolicity of $0.5 \mathrm{eV}^{-1}$ [11]. One type of acoustic phonons in the elastic approximation and equipartition is accounted for the electron intravalley scattering. The value of $9 \mathrm{eV}$ is used for acoustic deformation potential [11]. The intervalley scattering is modeled using a set of six large-momentum phonons [12]. We calculated the drift velocity amplitudes and phases taking the electric field of the fundamental wave along the principal directions in the crystal. Figure 1 shows an example of the drift velocity first and third harmonic amplitude and phase dependence on the fundamental wave field amplitude.

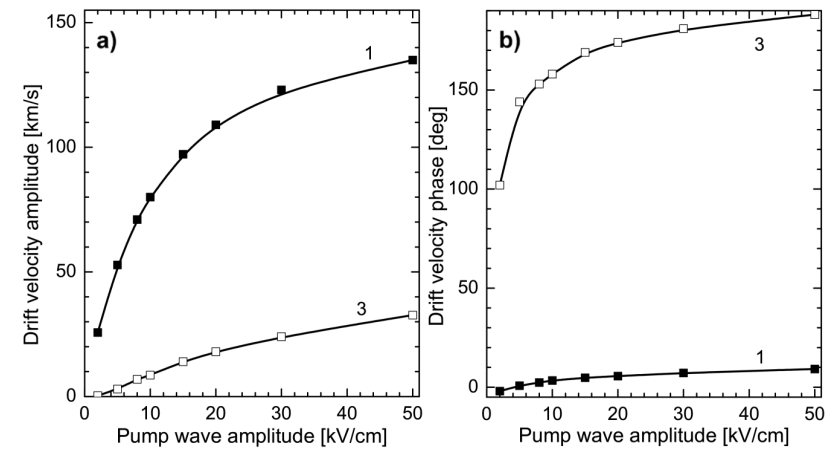

Fig. 1. Drift velocity amplitude (a) and phase (b) of the first (1) and third (3) harmonics in $950 \Omega \mathrm{cm} n$-type $\mathrm{Si}$ as a function of the electric field amplitude at the frequency of $118 \mathrm{GHz}$ and the temperature of $300 \mathrm{~K}$. The electric field of the pump wave is parallel to the $\langle 111\rangle$ direction of the crystal.

The amplitudes and phases of the drift velocity harmonics tend to saturate at high pumping wave fields. Similar behavior is observed for other principal directions [9]. The anisotropy of the third order nonlinearity is most pronounced at low pumping wave fields (Fig. 2).

With the rise of the pump wave field amplitude the square of the absolute value of the 3rd order susceptibility reduces dramatically and the anisotropy becomes negligible.

More informative is the ratio of the 3rd order component of the drift velocity amplitude to the pump wave field amplitude that we refer to as the THG electronic yield (Fig. 3). It is a maximum at certain range of pump wave amplitude, showing a guideline for the $3 \mathrm{rd}$ harmonic generation efficiency optimization. Compared to the other principal directions, in $\langle 111\rangle$ direction the electronic yield exhibits a maximum at higher pumping wave fields.

The THG efficiency is defined as the ratio of the emitted intensity $\left(I_{3}\right)$ to that of the incident pump wave $\left(I_{1}\right)$.

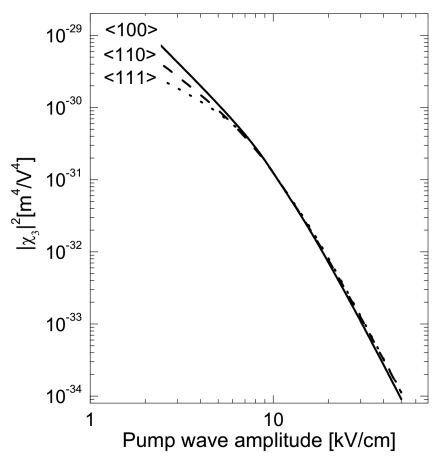

Fig. 2. Anisotropy of the square of the third order non-linear susceptibility in $950 \Omega \mathrm{cm} n$-Si crystal, obtained from the Monte Carlo simulations at $118 \mathrm{GHz}$ and $T=$ $300 \mathrm{~K}$.

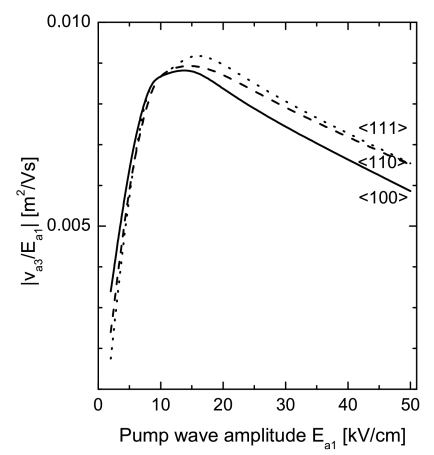

Fig. 3. Anisotropy of the electronic yield in $950 \Omega \mathrm{cm}$ $n$-Si crystal, obtained from the Monte Carlo simulations at $118 \mathrm{GHz}$ and $T=300 \mathrm{~K}$.

With the assumption, that the electron contribution in (4) and (5) is small, for thin slabs it can be expressed as [9]:

$$
\eta_{3}=\frac{I_{3}}{I_{1}} \propto N^{2} e^{2} d^{2}\left|\frac{V_{a 3}}{E_{a 1}}\right|^{2} .
$$

Here $d$ stands for the slab thickness. Thus, if the surface carrier density $N d$ is constant, the THG efficiency is determined by the square of the electronic yield.

The THG efficiency is evaluated conventionally assuming the sample refraction index and the nonlinear susceptibility to be constant over the whole sample. Actually the values are controlled by the electron plasma contribution that is amplitude-dependent due to electron heating. The change of the wave amplitude inside the crystal modifies the amplitude and phase relations between the 1st and 3rd harmonic beams. The new feature of theory in this work implies appropriate description of the experimental 3rd harmonic generation on the basis of wave interaction with electron plasma, including also the refractive index modulation effects. It is the matter of considerations presented below (Sect. 4).

\section{Experiment}

THG has been measured on the $n$-type Si crystals with the specific resistance of $\rho_{0}=950 \Omega \mathrm{cm}$ and diameter 
$7.6 \mathrm{~cm}$. Parallel plates of $5.4 \mathrm{~mm}, 10.8 \mathrm{~mm}, 21.45 \mathrm{~mm}$ and $43 \mathrm{~mm}$ thickness were stacked to vary the length of nonlinear interaction. The pump wave in form of a $\mathrm{HE}_{11}$ mode was generated by $118 \mathrm{GHz}$ gyrotron with the output power up to $500 \mathrm{~kW}$ and $2 \mathrm{~ms}$ pulse length. The beam was focused into the $12 \mathrm{~mm}$ spot at the waist position using 2 spherical mirror set (Fig. 4). The broad-band pyroelectric detector $(0.001-1000 \mu \mathrm{m})$ with the sensitivity of $\approx 5 \mathrm{mV} / \mathrm{W}$ at the 3rd harmonic frequency was placed inside the Faraday cage. To avoid the fundamental frequency detection the high-pass filter was installed in front of the detector. The filter transmission measurements have confirmed the fundamental wave attenuation at least to $70 \mathrm{~dB}$, and negligible attenuation at the $3 \mathrm{rd}$ harmonic. The leaking power $\approx 1.4 \mathrm{~W}$ was detected without the Si sample. Its origin is not readily known but the radiation can arise, e.g., in the gyrotron windows. The 3rd harmonic power emitted by the Si sample was considerably higher.

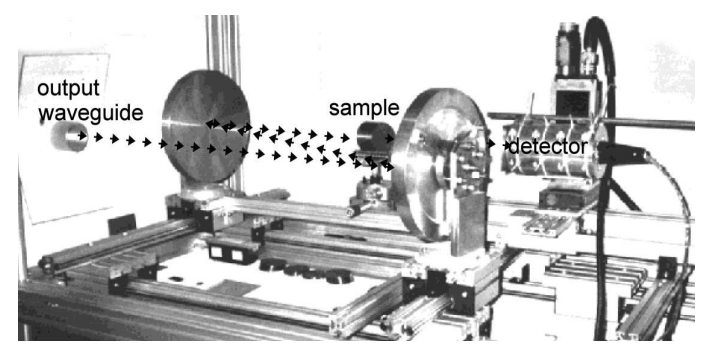

Fig. 4. Experimental setup used for 3rd harmonic generation experiment. Arrows mark the gyrotron beam propagation direction.

First experiment was performed in the Brewster geometry. In order to suppress the reflection from the interfaces and increase the THG efficiency, in this geometry pumping wave was polarized in the plane of incidence ( $p$-polarization) and the angle of incidence was equal to the Brewster angle. Samples were cut on the angle, which ensured the beam propagation along the axis of the sample, parallel to the $\langle 100\rangle$ direction. We investigated the dependence of the THG detector signal on the sample thickness. The 3rd harmonic beam intensity distribution was measured using the manipulator with step-size of $10 \mu \mathrm{m}$. The horizontal detector position was optimized and kept during the rest of experiments, and the vertical one scanned for the every set of the samples. With increased sample thickness we observed monotonic down-shift and increase of the third harmonic power (Fig. 5). When thickness exceeded $7 \mathrm{~cm}$, the third harmonic power decreased dramatically.

In the next experiment the fundamental wave incident at the angle of $16.4 \mathrm{deg}$ was polarized normally to the plane of incidence ( $s$-polarization), so the directions of the 1st and 3rd harmonic propagation were non-collinear because of the refraction index dispersion. This geometry was therefore called "non-collinear". While in this configuration a part of the incident power was reflected, the

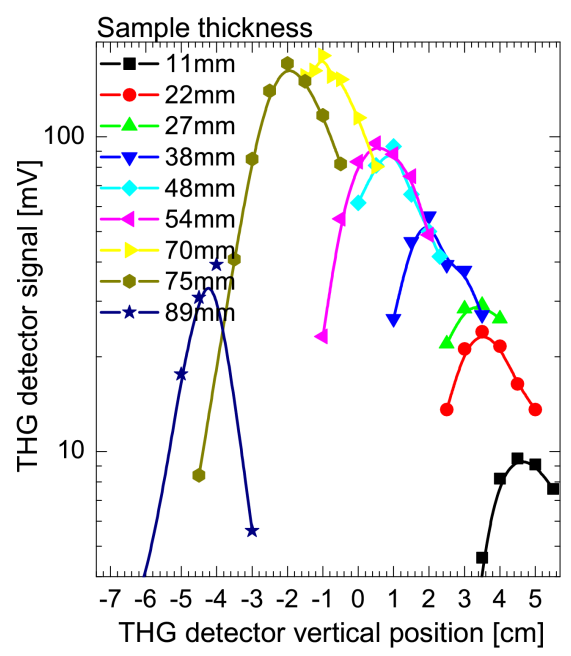

Fig. 5. The 3rd harmonic signal observed in the Brewster geometry for different thickness of $n$-type Si crystals, $T=300 \mathrm{~K}$. Distances: detector-filter: $5 \mathrm{~cm}$, filtersample: $40 \mathrm{~cm}$, sample-mirror: $50 \mathrm{~cm}$ (at the focal distance).

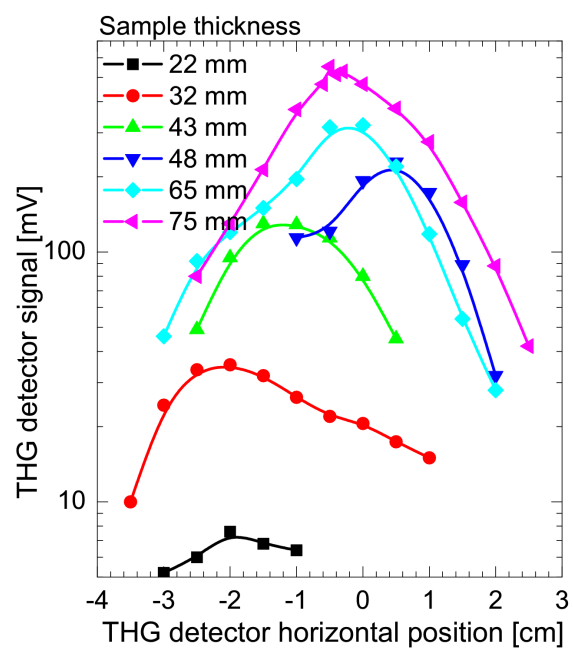

Fig. 6. The 3rd harmonic signal observed in the non-collinear geometry for different thickness of $n$-type Si crystals, $T=300 \mathrm{~K}$. Distances: detector-filter: $5 \mathrm{~cm}$, filter-sample: $42 \mathrm{~cm}$, sample-mirror: $50 \mathrm{~cm}$ (at the focal distance).

electrical field amplitude in the sample was still higher than in the Brewster geometry, resulting in the higher third harmonic generation efficiency.

Figure 6 illustrates the power distribution observed when the vertical position of the detector is optimized and fixed, and the horizontal is varied across the beam. The distribution turns out to be quite different from the fundamental-wave Gaussian distribution. Besides the sharpened central part of the intensity profile, the double beams are observed in the emitted 3rd harmonic. Some errors could arise from the fundamental wave power fluctuations from shot to shot. 

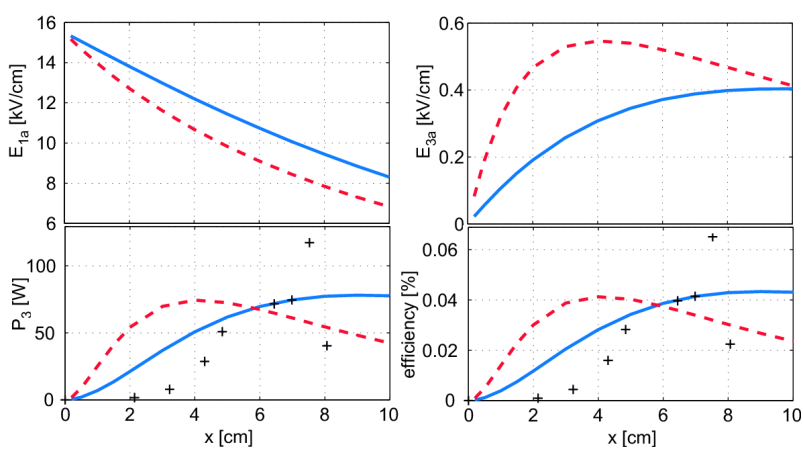

Fig. 7. The fundamental wave electric field $\left(E_{1 a}\right)$, and the 3rd harmonic field $\left(E_{3 a}\right)$, and the power $\left(P_{3}\right)$ distribution over the distance $x$ from the beam input face in the $10 \mathrm{~cm}$ thick $n$-type Si slab. Blue (solid) lines: calculated taking into account free carrier system response to the high electric field of the pumping wave; red (dashed) lines: calculated for the small-signal values of $\varepsilon_{1}$ and $\varepsilon_{3}$, supposing the constant value of $\chi_{3}=5 \times 10^{-16} \mathrm{~m}^{2} / \mathrm{V}^{2}$. The incident pump wave power is $180 \mathrm{~kW}$. Crosses: experimental data obtained for the set of slab thickness values.
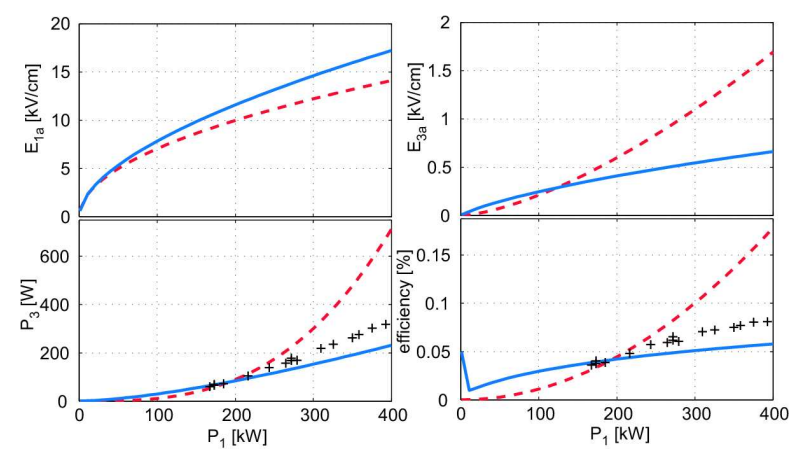

Fig. 8. The fundamental wave electric field $\left(E_{1 a}\right)$, and the 3rd harmonic field $\left(E_{3 a}\right)$ and power $\left(P_{3}\right)$ dependence on the incident pump wave power $\left(P_{1}\right)$. Blue (solid) lines: calculated taking into account free carrier system response to the high electric field of the pumping wave; red (dashed) lines: calculated for the small-signal values of $\varepsilon_{1}$ and $\varepsilon_{3}$, supposing the constant value of $\chi_{3}=5 \times$ $10^{-16} \mathrm{~m}^{2} / \mathrm{V}^{2}$; crosses - experimental data for the slab thickness of $65 \mathrm{~mm}$.

The THG power dependence on the sample thickness was studied (Fig. 7). In both investigated geometries the 3rd harmonic intensity has decreased dramatically when the sample thickness exceeded $7 \mathrm{~cm}$; the switching effect was limiting the feasible THG efficiency. The 3rd harmonic power dependence on the fundamental one (Fig. 8) did not follow the cubic law, and the saturation was observed at highest pumping levels. The maximum achieved THG power was $\approx 300 \mathrm{~W}$ at $400 \mathrm{~kW}$ of pumping wave showing the efficiency slightly above $0.07 \%$. Arcing at the Si surface prevented further pumping wave power increase.

\section{Discussion}

According to an ideal model of THG, the emitted power grows as the cube of the pump power, promising high-efficiency of frequency tripling at sufficiently intense pumping in the direct (single-pass) experiments. Actually, THG power saturation is observed in experiments. It is explained on the basis of numerical calculations of the self-adjusted spatial distributions of electron drift velocity and the pumping wave electric field amplitudes [9]. The amplitude and phase of the Fourier components of electron drift velocity dependence on the fundamental wave electric field amplitude is used to calculate the electronic part of the linear and nonlinear susceptibility.

We solve the wave Eq. (1) in the dynamically non-uniform Si slab, where the first and third order susceptibilities depend on the pumping wave amplitude. The latter is subject to substantial change due to absorption in the slab. The slab is divided into small layers where the dielectric functions and the nonlinear susceptibility can be approximated by constant values. The initial fundamental wave electric field distribution in the slab is calculated using the thickness- (and electric field-) independent complex values $\varepsilon_{1}, \varepsilon_{3}$ for the whole sample. They can be obtained from experimentally measured absorption coefficient $\left(\alpha=0.1 \mathrm{~cm}^{-1}\right)$ and lattice dielectric constant $\left(\varepsilon_{\mathrm{L}}=11.69\right)$. Then the fundamental wave field profile in the slab is generated. This profile is used to obtain thickness-dependent dielectric functions $\varepsilon_{1}, \varepsilon_{3}$ (Fig. 9) and the susceptibility $\chi_{3}$ (Fig. 10). The inhomogeneous $\mathrm{Si}$ slab can now be approximated by the layered medium.

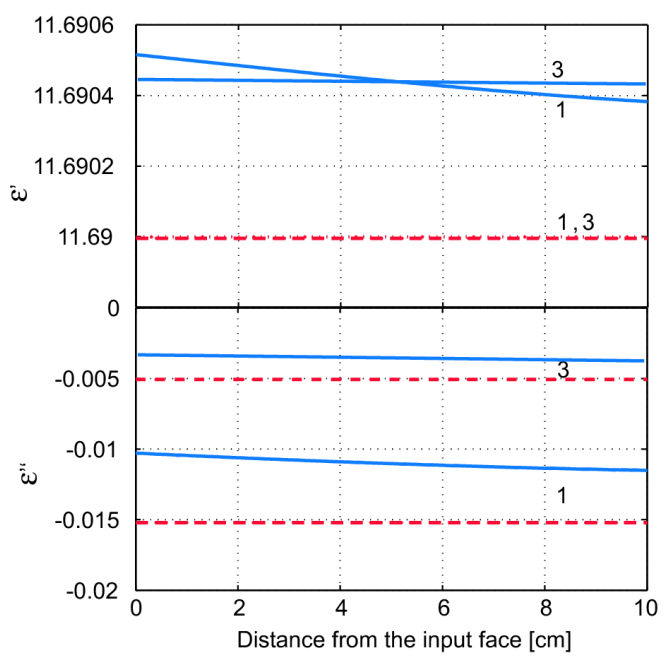

Fig. 9. Real $\left(\varepsilon^{\prime}\right)$ and imaginary $\left(\varepsilon^{\prime \prime}\right)$ parts of the dielectric function depending on the coordinate along the beam direction in the $10 \mathrm{~cm}$ slab of $n$-type Si. Solid (blue) lines: calculated taking into account free carrier system response to the high electric field of the pumping wave; dashed (red) lines - small-signal values. The field amplitude distribution is shown in Fig. 7. Numbers 1 and 3 denote the first and third harmonics. 


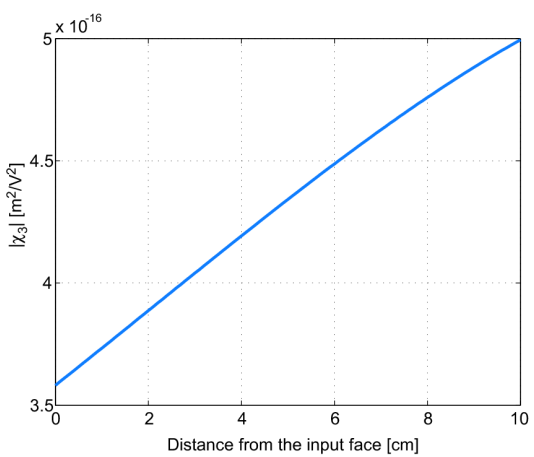

Fig. 10. The third-order susceptibility distribution over the $10 \mathrm{~cm}$ slab thickness of $n$-type Si accounting for the field amplitude profile shown in Fig. 7.

The homogeneous wave equation for the 1st harmonic is then solved by the matrix method [13]. The same method is used for the 3rd harmonic nonlinear wave Eq. (1). It should be just applied not only to the eigenmodes (homogeneous solution), but also to the source (polarization) wave. The latter can be obtained from Eq. (2) using known fundamental wave amplitude inside of each artificial layer.

The 1st harmonic electric field amplitude dependence on the sample thickness for the uniform $n$-Si slab is stronger than that for the layered medium model (Fig. 7). As a result, the 3rd harmonic electric field in uniform $n$-Si slab approaches maximum for thinner samples than in the case of the layered medium. As one can conclude from the comparison with the experimental data, the 3rd harmonic power and efficiency dependence on the layer thickness for the layered medium model is much more realistic. But it also cannot explain dramatic power rise and then drop for thick samples. The reason could be the plane wave model limitations. The confocal Gaussian beam parameter $b$ is in our case comparable with sample thickness, whereas the plane wave approximation holds when $b \gg d$. The switching phenomenon arises likely due to violation of the 1st and 3rd harmonic phase velocity synchronism that is controlled by the local value of pump wave amplitude. To obtain the correct value of this amplitude, the wave front curvature should be taken into account.

The same layered medium model is applied to explain the THG dependence on the fundamental wave power (Fig. 8). Here the constant susceptibility approximation leads to the cubic law, which strongly overestimates THG power and efficiency at higher pumping power. If $\varepsilon_{1}, \varepsilon_{3}$ and $\chi$ dependences on fundamental wave power are taken into account, the theoretical curve is much closer to the experimental data. The efficiency saturates at highest power levels. That effect is slightly overestimated by the layered medium model.

\section{Conclusions}

THG peak efficiency in $n$-type $\mathrm{Si}$ (with the specific resistance of $950 \Omega \mathrm{cm}$ at $T=300 \mathrm{~K}$ ) measured at the pump wave frequency of $118 \mathrm{GHz}$ is $0.07 \%$. Measurements at two configurations have revealed that the Brewster geometry gives rise to less efficient generation compared to the case of the non-collinear geometry. The efficiency increases monotonically with the rise of the sample thickness up to a threshold value, and decreases dramatically above the threshold. Except of this switching effect, the 3rd harmonic generation is reasonably describable within the model of drift non-linearity of electrons driven by high-amplitude electric field.

The drift non-linearity model based on the Monte Carlo calculations is applied together with the solutions of non-linear wave equation for the single pass through the sample. The theory accounting for the dynamic non-uniformity arising due to the spatial change of the pump wave amplitude gives the efficiency value that is in reasonable agreement with experiments although there remains unexplained switching effect and the non-Gaussian shape of THG beam. The THG prediction method presented here can easily be applied to any other crystals exhibiting electron drift nonlinearity.

\section{References}

[1] J.P. Feve, B. Boulanger, Y. Guillien, Opt. Lett. 25, 1373 (2000).

[2] K. Miyata, N. Umemura, K. Kato, Opt. Lett. 34, 500 (2009).

[3] F. Gravier, B. Boulanger, Opt. Express 14, 11715 (2006).

[4] M. Siegrist, F. Keilmann, C. Nieswand, M. Urban, Infrared Phys. Technol. 36, 407 (1995).

[5] F. Keilmann, R. Brazis, H. Barkley, W. Kasparek, M. Thumm, V. Erckmann, Europhys. Lett. 11, 337 (1990).

[6] M. Urban, Ph.D. Thesis No. 1492, EPFL, Lausanne 1996.

[7] N. Blombergen, P.S. Pershan, Phys. Rev. 128, 606 (1962).

[8] M. Urban, M.R. Siegrist, L. Asadauskas, R. Raguotis, R. Brazis, Appl. Phys. Lett. 69, 1776 (1996).

[9] R. Brazis, R. Raguotis, M.R. Siegrist, J. Appl. Phys. 84, 3474 (1998).

[10] C. Jacoboni, P. Lugli, The Monte Carlo Method for Semiconductor Device Simulation, Springer, Wien 1989.

[11] A. Dargys, J. Kundrotas, Handbook of Physical Properties of Ge, Si, GaAs and InP, Science and Encyclopedia Publ., Vilnius 1994.

[12] R. Bruneti, C. Jacoboni, T. Nava, L. Reggiani, G. Bosman, R.J.J. Zijlstra, J. Appl. Phys. 52, 6713 (1981).

[13] P. Yeh, Optical Waves in Layered Media, Wiley, New York 1988 